\title{
DELIRIUM AND DEMENTIA
}

Delirium is defined as a transient global disorder of cognition. Many factors lead to delirium including baseline vulnerability interacting with precipitants during hospitalization. Delirium affects an estimated 2.3 million hospitalized elders annually, accounting for 17.5 million inpatient days, and leading to more than $\$ 4$ billion in Medicare costs. It is associated with increased mortality, high rates of functional and cognitive decline, prolonged lengths of stay and high rates of skilled nursing facility placement. The cost of caring for patients with delirium significantly impacts individual patients, families and hospital systems, and accounts for billions of the Medicare budget. Hospitalists lead their institutions in the development of screening and prevention protocols for patients at risk for delirium, and in the promotion of safe approaches to treatment. Hospitalists also develop strategies to operationalize cost-effective delirium prevention programs that will improve outcomes.

Dementia is defined as a progressive decline in cognitive function, eventually limiting daily activities. Dementia is a common co-morbidity in the hospitalized elder. Patients with dementia are at increased risk for delirium, falls, and functional decline during hospitalization. Patients with baseline cognitive impairment have prolonged lengths of stay and complex needs after discharge. Agitation and behavioral symptoms of dementia can exacerbate and be difficult to manage in the hospital setting. Care of the patient with dementia requires that hospitalists engage in a multidisciplinary approach to inpatient and transitional care. Hospitalists may also become involved in hospital quality and safety initiatives that pertain to areas such as restraint use and fall prevention.

\section{KNOWLEDGE}

Hospitalists should be able to:

- Define delirium and dementia.

- Distinguish the causes of delirium.

- Describe the indicated tests required to evaluate delirium and dementia.

- Recognize innate and environmental/iatrogenic risk factors for the development of delirium in the hospitalized patient.

- Identify medications known to precipitate delirium.

- Explain indications, contraindications and mechanisms of action of pharmacologic agents used to treat delirium and dementia.

- Determine the best setting within the hospital to initiate, monitor, evaluate and treat patients with delirium.

- Describe the poor outcomes related to delirium and dementia in the hospitalized patient.

- Explain goals for hospital discharge, including specific measures of clinical stability for safe care transition.

\section{SKILLS}

Hospitalists should be able to:

- Distinguish delirium and dementia from other causes of cognitive impairment, confusion or psychosis.

- Predict a patient's risk for the development of delirium or poor outcomes related to dementia based on initial history and physical examination.

- Screen for delirium using appropriate testing early and repeatedly during the patient's hospital course.

- Perform a screen for dementia using the appropriate testing.

- Apply known patient risk factors to create a care plan for reducing delirium.

- Perform a focused evaluation for the underlying etiology of delirium and institute prompt treatment to lessen the severity of delirium.

- Formulate and lead multidisciplinary teams to develop and implement care plans for patients with delirium or dementia.

- Prescribe appropriate medications and dosing regimens for patients with delirium or dementia.

- Repeatedly assess the need for additional interventions.

- Assess patients with suspected delirium in a timely manner, identify the level of care required, and manage or co-manage the patient with the primary requesting service. 


\section{ATTITUDES}

Hospitalists should be able to:

- Communicate with patients and families to explain the history and prognosis of delirium or dementia.

- Communicate with patients and families to explain goals of care plan, discharge instructions and management after release from hospital.

- Educate and engage families in the care of elder inpatients.

- Establish goals and boundaries of care with patients and their family.

- Communicate with the families and others with durable powers of attorney to explain tests and procedures and their indications, and to obtain informed consent.

- Recognize the indications for specialty consultations.

- Describe methods for the prevention of delirium.

- Employ a multidisciplinary approach to the care of patients with delirium or dementia that begins at admission and continues through all care transitions.

- Responsibly address and respect end of life care wishes for patients with advanced dementia.

- Realize the multi-faceted impact of delirium or dementia on patients and their families.

- Appreciate and document the value of appropriate treatment in reducing mortality, duration of delirium, time required to control agitation, adequate control of delirium, treatment of complications, and cost.

- Facilitate discharge planning early in the hospitalization, including communicating with the primary care provider, and presenting the patient and family with contact information for follow-up care, support and rehabilitation.

- Utilize evidence based recommendations to guide diagnosis, monitoring and treatment of delirium and its causes.

\section{SYSTEM ORGANIZATION AND IMPROVEMENT}

To improve efficiency and quality within their organizations, Hospitalists should:

- Lead multidisciplinary teams to develop early treatment protocols.

- Lead, coordinate or participate in multidisciplinary initiatives to implement screening and prevention protocols for patients at risk for delirium or poor outcomes related to dementia.

- Engage stakeholders in hospital initiatives to improve safety and quality in the care of delirious and demented patients (e.g. provide diversion activities rather than using restraints).

- Lead, coordinate or participate in multidisciplinary initiatives, which may include collaboration with

- geriatricians, to promote patient safety and cost-effective diagnostic and management strategies for elderly patients. 